\title{
Aspectos fundamentales de la complejidad y transdisciplinariedad en la educación superior
}

Dra. Carolina Serrano Barquín

carolinasb@hotmail.com

Mtra. Teresa Jazmín Flores Pérez

jaz2504@hotmail.com

Dra. Tania Morales Reynoso

taniamreynoso@gmail.com

Dra. Brenda Mendoza González

brenmx@yahoo.com.mx

Facultad de Ciencias de la Conducta

Universidad Autónoma del Estado de México

Recibido: 23 de enero 2020

Aceptado: 15 de febrero 2020

\section{Resumen}

La educación superior debe estar orientada al logro de las capacidades que se requieren para el desarrollo democrático, social y económico de cada país, entre otros fines, ya que actualmente, esto adquiere una dimensión estratégica dentro del ámbito del conocimiento. Con el advenimiento de redes, telefonía y modelo digital, la educación se reconfigura y aporta nuevas opciones. Por ello, es fundamental definirla desde todos sus ángulos, tomando en cuenta a todos sus actores y situaciones. Analizar y replantear sus objetivos, se convierte en una necesidad ante la diversificación de los recursos de los procesos de enseñanza y aprendizaje. La tendencia de los estudios actuales sobre cualquier problemática social es el abordaje multi e interdisciplinario, necesario para hacer una intervención capaz de disgregar cada uno de sus aspectos, sin olvidar la importancia de la transdisciplinariedad. Por otra parte, la perspectiva de la complejidad exige tomar en cuenta los factores que intervienen, así como sus causas y efectos; se trata pues, de un análisis consciente de todos los elementos. Sin duda, el pensamiento complejo es el espacio donde pueden coincidir las diferentes áreas para avanzar hacia el conocimiento; para ello, es necesario pensar en la complementariedad de las destrezas cognitivas como el razonamiento y el análisis que nos permite llegar al diálogo y a la metacognición, ya que no sólo se potencializa el conocimiento científico, sino también el razonamiento filosófico y humanístico. Ése debiera plantearse como el nuevo eje de la Educación Superior. Pensar el ámbito educativo desde la complejidad representa la búsqueda 
de programas que permitan reflexionar la realidad desde una perspectiva integral, de la unidad y de sus partes, y así evitar caer en un pensamiento reduccionista. Sólo así, el hombre será capaz de asumirse como parte de un tejido social y no sólo como individuo.

Palabras clave: Enseñanza superior, transdisciplinariedad, complejidad.

\title{
Fundamental aspects of complexity and transdisciplinarity in higher education
}

\begin{abstract}
Higher education must be oriented towards the achievement of the capacities that are required for the democratic, social and economic development of each country, among other purposes, since currently, this acquires a strategic dimension within the field of knowledge. With the advent of networks, telephony and digital model, education is reconfigured and brings new options. Therefore, it is essential to define it from all angles, taking into account all its actors and situations. Analyze and rethink its objectives, it becomes a necessity in view of the diversification of the resources of the teaching and learning processes. The tendency of current studies on any social problem is the multi and interdisciplinary approach, necessary to make an intervention capable of disaggregating each of its aspects, without forgetting the importance of transdisciplinarity. On the other hand, the perspective of complexity requires taking into account the factors involved, as well as their causes and effects; It is therefore a conscious analysis of all the elements. Undoubtedly, complex thinking is the space where different areas can coincide to move towards knowledge; For this, it is necessary to think about the complementarity of cognitive skills such as reasoning and analysis that allows us to reach dialogue and metacognition, since not only scientific knowledge is potentiated, but also philosophical and humanistic reasoning. That should be considered as the new axis of Higher Education. Thinking the educational field from complexity represents the search for programs that allow us to reflect reality from an integral perspective, of unity and its parts, and thus avoid falling into a reductionist thought. Only in this way will man be able to assume himself as part of a social fabric and not only as an individual.
\end{abstract}

Keywords: Higher education, transdisciplinarity, complexity. 


\section{Introducción}

Cuando se habla de educación, frecuentemente se recurre a plantear retos y necesidades, pareciera que existe una urgencia por resarcir las fallas operativas para seguir sosteniendo que la educación es el gran motor del desarrollo. Reforma, acuerdo, convenio o como sea que -coyunturalmente- convenga llamarle, se trata finalmente de una panacea temporal de los males globales.

Las prácticas educativas en el mundo son tan diversas como las formas de interacción humana; en ellas se imprimen valores, costumbres, formas de actuar y de concebir la vida. El contexto económico, social, político y cultural de cada país determina la producción del conocimiento. En este sentido, la sociedad espera que sus instituciones educativas contribuyan en la mayor medida de lo posible a generar propuestas y alternativas reales a los problemas que enfrenta.

Alcanzar una educación pertinente y de calidad no debe ser sólo un propósito, sino un proyecto que incluya a todos los sectores, y para ello es necesario analizar su status quo y sus oportunidades de desarrollo. Un estudio integral abre las posibilidades de descubrir vías de solución alternas, así como nuevos medios y herramientas para mejorar los procesos al hacerlos más eficientes.

Probablemente, el principio del análisis de la educación se encuentre en los paradigmas; en el conjunto de perspectivas, reflexiones y experiencias mediante las que se aborda el fenómeno educativo. Generalmente, estos paradigmas desarrollan aspectos como la concepción, los objetivos y la organización de la educación, proporcionando fundamentos explicativos desde enfoques diversos que han sido retomados durante décadas como modelos teóricos que sustentan una cantidad infinita de investigaciones sobre los procesos de enseñanza y de aprendizaje y, ¿cómo se estudia el caos que lo envuelve?

El desorden forma parte de la realidad y, por lo tanto, de la construcción del conocimiento; los problemas de individuos y sociedades se vuelven cada vez más complejos: característica principal del mundo globalizado. Mientras que, por un lado, se fomenta la evolución tecnológica, por otro, crece la insensibilidad y la deshumanización; se vuelve fundamental desarrollar habilidades para entender el mundo complejo e intervenir en él. 
Dicho lo anterior, es el momento de afirmar que los problemas actuales son esencialmente transdisciplinares y totalmente complejos. Transdisciplinares porque trascienden la explicación que se les puede dar desde cada una de las disciplinas que se han abocado a estudiar uno sólo de sus aspectos. Complejos porque ya no es posible acotar su análisis a uno sólo de sus ángulos. Cuando comienzan a explorarse los aspectos físicos, biológicos y culturales del mundo en el que nos desarrollamos, es a nosotros mismos a quienes descubrimos.

Transdisciplina y complejidad son formas de pensamiento que se conectan entre sí para abordar la vida humana y social. Según Nicolescu (s.a.), volvemos entonces a la imperiosa necesidad de proponer, vivir, aprender y enseñar un pensamiento complejo, que vuelva a tejer las disciplinas como posibilidad de humanidad en completud; y que sólo de esta manera se vencería la eterna limitación y fragmentación del sujeto separado de sí mismo en la búsqueda del conocimiento. Una pedagogía transdisciplinaria (Pérez, Moya y Curcu, 2013), debe abocarse al ser del que aprende a través de una formación atravesada por la lógica, la ética y la estética. Debe dar cuenta de cómo establecer nexos entre los conocimientos especializados que se estancan al no conectarse y cómo transversar éstos con los otros campos disciplinares.

El estudio de cualquier aspecto de la experiencia humana, señala Morin (1990) ha de ser, por necesidad, multifacético; pues la mente humana no existe sin tradiciones familiares, sociales, genéricas, étnicas y raciales; todo aquello que hemos llamado cultura. Lo cierto es que mientras más se conoce de ella, existe un mayor acercamiento al pensamiento reduccionista que restringe la experiencia a sectores limitados del saber.

\section{Estado actual de la educación superior}

La educación superior es riqueza de índole estratégica para las naciones; Hugo Rangel (2015) en su texto Educación superior sin ataduras: College (Un) Bound, concibe a la educación superior como aquella educación que por sí misma mejora la calidad de vida y ayuda a darle significado a la de quienes estudian, que a partir de ella, se vive mejor y más sanamente. El conservadurismo rechaza estas cualidades, lo que amerita presentar mayores argumentos en 
razón de los cuestionamientos ideológicos, las presiones sobre las universidades y los problemas sociales.

Esta aportación se centra en una reflexión filosófica que recupera el aspecto vocacional, que resulta esencial en la tarea educativa. La labor del profesional y su impacto constituyen el sentido de su vida y su razón de ser dentro de la estructura social, así como su devenir como ser humano con su desempeño profesional.

En una perspectiva más pragmática, el Programa Sectorial de Educación 2013-2018 de la Secretaría de Educación Pública, señala que la educación superior debe estar orientada al logro de las competencias que se requieren para el desarrollo democrático, social y económico del país. Es en la educación superior que cada estudiante debe lograr un sólido dominio de las disciplinas y valores correspondientes a las distintas profesiones. "La diversificación del sistema de educación superior y su amplia presencia en las distintas regiones son condiciones que favorecen la pertinencia de la educación superior, para hacer una contribución creciente al mejoramiento social y el aumento en la productividad necesario para mejorar la competitividad de la economía” (Programa Sectorial de Educación 20132018, p. 8).

En México, como en muchos otros países, la matrícula y la demanda, van en aumento; lo que obliga a poner sobre la mesa el tema de la calidad y las necesidades cambiantes de profesionalización. Las instituciones públicas enfrentan el desafío de una mayor cobertura con una variante mínima de presupuesto; lo cierto es que no se trata sólo de incrementar la matrícula, sino de prevenir que su masificación repercuta en la calidad de los programas educativos.

En 2009, la Conferencia Mundial sobre la Educación Superior de la Organización de las Naciones Unidas para la Educación, la Ciencia y la Cultura (UNESCO), consensó que los principales retos de este nivel educativo en el siglo XXI se concentran en la comprensión de los complejos fenómenos sociales contemporáneos y en la capacidad para afrontarlos. Esto significa que la educación superior es el campo ideal para estudiarlos detalladamente y participar de las soluciones más convenientes desde la docencia, la investigación, la difusión de la cultura y el avance tecnológico. 
Visto así, la educación superior es una inversión necesaria, un pilar que vale la pena fortalecer para enriquecer la cultura con la contribución de las humanidades, las artes, la ciencia y tecnología; y para mejorar la competitividad en la economía del conocimiento, también es un agente impulsor de la justicia y la cohesión social. El pensamiento de Paulo Freire (Serna, 2016), considera que la educación es un proyecto profundamente político, orientado hacia la transformación de la sociedad y ha sido crucial para la educación de las sociedades modernas, así como en las democracias occidentales. Su teoría pedagógica ha ejercido una influencia considerable entre los educadores en gran parte del mundo, especialmente en el contexto de las tradiciones emergentes de la transdisciplinariedad, la pedagogía crítica, la educación continuada y la educación multicultural, entre otras.

Por otro lado, en el mundo contemporáneo, la diversidad cultural adquiere una nueva dimensión, no sólo por la interconexión de un mundo globalizado, sino por la necesidad de voltear la mirada hacia nuevos grupos, lo que supone superar los estereotipos y prejuicios sociales. La multiculturalidad en la educación superior, como en todos los niveles, es un fenómeno creciente en el que personas de diferentes grupos con características y prácticas particulares, conviven en un mismo espacio.

Este factor, exige un análisis crítico de los planteamientos pedagógicos para avanzar hacia un proyecto integrador, capaz de potenciar el intercambio de saberes y experiencias y así, superar la idea de que la multiculturalidad es sólo un concepto indicador de diferencias. Lo anterior es una tarea fundamental que conmina a todos los involucrados, pero sin duda, el profesor, tutor o asesor, es una importante figura de la mediación cultural.

Los docentes han dejado de ser sólo transmisores de contenidos, hoy en día, el profesor debe trabajar en pro de la autonomía; procurar que los educandos aprendan por su propia cuenta mediante un proceso continuo de socialización con los distintos grupos humanos para motivar al estudiante a descubrir sus capacidades, explotar sus habilidades y alcanzar sus propósitos; también es quien inicia la producción académica y promueve la práctica de los valores éticos. Los temas susceptibles de debate en la educación son inagotables; desde las cuestiones humanas y filosóficas, hasta las políticas y económicas. 
Aunque abordar todos los elementos que conforman el tema educativo de manera particular es posible, pero no será lo más útil.

\section{Transdisciplinariedad}

Durante mucho tiempo se pensó que la especialización de las disciplinas era lo más conveniente, que debían mantener distancia entre ellas para conservar sus cualidades, entonces, más tarde fue necesario admitir que se complementan entre sí, para dar paso a la multidisciplinariedad y posteriormente a la interdisciplinariedad, en la que los diferentes saberes trabajan entre sí para construir un conocimiento más robusto.

María Moraes (2007), comenta que la transdisciplinariedad es la que nos invita a trascender la relación binaria A y no-A, y observar que la relación es multifactorial y que no hay elementos contradictorios, sino complementarios. Es la que también nos lleva a transgredir y a romper las fronteras disciplinares, reconociéndolas no como barreras, sino como nuevos territorios que serán explotados, como espacios de trueque, de intercambios, de enriquecimiento mutuo, que permiten nuevas miradas al conocimiento.

El planteamiento de la transdisciplinariedad es generar pluralidad en el conocimiento, producir el diálogo entre ciencia y humanidad, entre saber académico y saber popular, que proviene de otras culturas y confrontar el conocimiento científico con otros conocimientos, para entonces, enlazar estos diferentes saberes y la vida ordinaria.

Para establecer la relación entre educación y transdisciplina es necesario partir de un concepto ordenador que permita explicar las implicaciones pedagógicas del proceso de transversalidad como elemento base del diálogo de saberes, según explican Pérez, Moya y Curcu (2013). Asimismo, mencionan que lo transdisciplinario, desde una mirada educativa, debe incorporar una búsqueda que no se restrinja a lo disciplinar, sino que conciba al saber y sus relaciones desde la idea de la totalidad como una forma de pensar lo real, así se produce una nueva interpretación de los conceptos, porque la realidad no es estática, sino que se despliega en su propio movimiento histórico. Es decir, el proceso educativo permite, a través del diálogo de saberes, un pensar transversal que a su vez implique la búsqueda en lo transdisciplinar. 
El concepto ordenador (reemplazo de lo teórico general por una pretensión epistemológica), permite pensar la realidad desde otras perspectivas. Esto es, establecer una relación de posibilidad entre los conceptos y avanzar en la especificación de sus contenidos mediante la reconstrucción de la vinculación.

La mirada transdisciplinaria, desde el punto de vista pedagógico, se entiende como un giro global al interior de la configuración del saber y el conocer; en este sentido, se puede hablar de trans saberes, ya que no es el saber académico institucionalizado y escolarizado el único que puede poseer "validez" epistemológica; por el contrario, el saber "irregular" o "extra académico" puede pensarse en conjunción para concebir una sinergia de saberes plurales, abiertos, creativos, móviles y flexibles que conduzcan a una expresión ampliada de la realidad. Lo transdisciplinario permite incorporar saberes subestimados por los cánones disciplinarios, pero no sólo saberes, sino identidades, formas de aprender, maneras de concebir el conocimiento, hibridaciones culturales, transmisión de experiencias, etc. (Pérez, Moya y Curcu, 2013, p.17).

El concepto ordenador (reemplazo de lo teórico general por una pretensión epistemológica), permite pensar la realidad desde otras perspectivas. Esto es, establecer una relación de posibilidad entre los conceptos y avanzar en la especificación de sus contenidos mediante la reconstrucción de la vinculación.

\section{Complejidad}

Antes, el conocimiento era duradero, pero ahora lo simplista, lo efímero y el consumismo, hacen una educación líquida, afirma Bauman (2008). En lo cotidiano, decir que algo es complejo resulta lo mismo que decir que es difícil, inaccesible, que carece de orden y que, por lo tanto, es complicado de explicar, de abordar y resolver. El término se ha estigmatizado, no se le concede el dinamismo y la energía que conlleva; complejidad refiere a la realidad que vive el ser humano cada día, no es necesario ser un científico para darse cuenta de que todo a nuestro alrededor es complejo. En la opinión de Pérez, Moya y Curcu (2013, p. 16), "la realidad se asume más allá de lo constituido, esto requiere destacar las opciones de explicaciones teóricas que a través del proceso de transversalidad la definen como múltiple y compleja". Los grandes descubrimientos que perduran hasta la actualidad, en su mayoría han sido resultado de procesos lineales, lógicos, basados en la experimentación, la comprobación, y la certidumbre; sin embargo, las formas de pensar y actuar evolucionan, y 
la humanidad se reconfigura a partir de éstas. ¿Y por qué no plantear la contraparte? En este sentido sería importante generar una conciencia sobre lo inesperado, estar preparados para cualquier acontecimiento; las sociedades se caracterizan por ser espontáneas e impredecibles, lo que conlleva desarrollar un pensamiento cuidadoso de los detalles, alerta de los procesos y crítico de su propio actuar.

Respecto a lo anterior, Edgar Morin señala que “...la conciencia de la complejidad nos hace comprender que no podremos escapar jamás a la incertidumbre y que jamás podremos tener un saber total: la totalidad es la no verdad" (Morin, 1990, p. 101). Si complejo es sinónimo de complicado, el argumento es más evidente: es necesario trascender estos conceptos para pensar tanto los elementos constitutivos como el todo.

Una de las principales críticas que hace este autor al sistema educativo, es la independencia de los contenidos, que no exista relación entre ellos, ni con la vida cotidiana. Lo anterior, refiere un ejemplo de la desarticulación en la que no se debe caer y de la necesidad de organizar los saberes de tal forma que no sólo sean complementarios, sino que puedan aplicarse a la realidad y sumen también a la visión transdisciplinaria.

\section{Conclusiones}

En su paso por sus responsabilidades, los diferentes actores del universo educativo; autoridades, profesores, alumnos, padres y madres de familia, constatan el funcionamiento real del aparato educativo, sus fortalezas y debilidades. Resulta imposible minimizar los problemas o reducirlos a cualquier variable, la realidad no se puede abordar unidimensionalmente, entonces su abordaje debe partir de la interrelación de todas sus variables. La complejidad es el estado natural de la sociedad en que vivimos actualmente.

La acción de educar, es decir, todos aquellos quehaceres y tareas que contribuyen a la formación de las personas, debe procurar que los involucrados antes mencionados, alcancen una visión holística, integradora y consciente del constante cambio y la incertidumbre. Que esta visión sea global, no quiere decir que sea desordenada, por el contrario, debe ayudar a que el individuo tenga una mayor claridad y juicio en sus ideas. 
Como sociedad, vivimos en una constante transformación motivada por la agilidad de los procesos, la rapidez de la información y las tendencias efímeras, lo que genera incertidumbre e inquietud. Es importante decir que nada de lo anterior merece un calificativo necesariamente negativo, la realidad es lo que percibimos y se conforma de todo lo acertado y lo inconveniente, de lo favorable y de lo desatinado. El reto es entonces, revolucionar las formas de abordar la educación superior para hacer un juicio realista, crítico y propositivo de ella, para entonces, generar otras formas de intervención, que ayuden no sólo a tener otras visiones, sino también a explicar situaciones alternas que definen su rumbo. Habrá pues, que reconocer otros saberes, no solo los académicos o científicos.

\section{Bibliografía}

Bauman, Z. (2008). Los retos de la educación en la modernidad líquida. Barcelona Gedisa.

Moraes, M. C. (2007): “Complejidad, transdisciplinariedad y educación: algunas reflexiones", Revista Encuentros Multidisciplinares nº 25, Vol. IX, pp. 4-13.

Morin, E. (1990). Introducción al Pensamiento Complejo. España: Gedisa Editorial.

Nicolescu, B. (s.a.). La transdisciplina. Manifiesto. Mónaco: Du Rocher.

Pérez Luna, Enrique; Moya, Norys Alfonzo y Curcu Colón, Antonio (2013). “Transdisciplinariedad y educación”, en Educere, vol. 17, núm. 56, enero-abril, 2013, pp. 15-26.

Programa Sectorial de Educación 20013-20018. Secretaría de Educación Pública, enero del 2014. Ciudad de México: Gobierno de la República Mexicana. http://normatecainterna.sep.gob.mx/work/models/normateca/Resource/253/1/images /programa_sectorial_educacion_2013_2018.pdf

Rangel, Hugo (2015). Educación superior sin ataduras: College (Un) Bound. Revista de la Educación Superior, Vol. XLIV núm. Enero-Marzo, pp. 189-194.

Santos Rego, M. (2000). El pensamiento complejo y la pedagogía. Bases para una teoría holística de la educación. Estudios Pedagógicos, (26), 133-148. 
Serna, E. (2016). "La transdisciplinariedad en el pensamiento de paulo Freire", en Revista de Humanidades, núm. 33, enero-junio, 2016, pp. 213-243.

Zabala, A. (1999). Enfoque globalizador y pensamiento complejo. Barcelona: Graó. 